\title{
A Survey on Machine Learning based Smart Maintenance and Quality Control Solutions
}

\author{
Attila Frankó ${ }^{1}$ and Pál Varga ${ }^{2}$
}

\begin{abstract}
Machine learning aided tasks and processes have key roles in smart manufacturing, especially in controlling production and assembly lines, as well as smart maintenance and intelligent quality control. The last two ones are those tasks that nowadays are still performed manually by employees; however, there are numerous machine learning-based solutions that can automate these fields to optimize cost and performance. In this paper, we present an overview of smart manufacturing ecosystem and define the roles of maintenance and quality control in it. Up-to-date machine learning-based smart solutions will also be detailed while addressing current challenges and identifying hot research topics and possible gaps.
\end{abstract}

Index Terms - machine learning, smart maintenance, intelligent quality control, diagnostics, health management, computer vision

\section{INTRODUCTION}

Over the last decade, the number of artificial intelligence (AI) based applications significantly raised, as certain emerging machine learning algorithms and technologies, such as deep learning (DL), gained popularity. Since then, machine learning has matured enough to enable its usage in various fields, from computer vision to machine-type communications. Its ability to solve complex, high-dimensional problems in a wide range made it one of the major drivers of the Industry 4.0 [1] movement besides industrial Internet of Things. As digitalization efforts are increasing within the industry to transform traditional manufacturing into highly automated smart factories, machine learning methods become the pillar of automating, not only manufacturing products within shop floors but maintenance tasks, logistical processes across the whole supply chain, warehouse management, automated quality management, production control - to mention the most popular ones [2].

Several global companies with sufficient technical and financial background have already applied machine learningbased solutions with success to automate certain tasks within warehouses or shop-floors, e.g., autonomous vehicles that can relocate assets without any manual interactions by operators.

Regardless of that, this area is still considered to be relatively new because of two factors: first, many areas within this field - such as automating the aforementioned processes and tasks - are not considered hot topics, despite that few of them (e.g. self-driving vehicles) dominate current research trends. Second, most market players - predominantly Small and Medium-sized Enterprises (SMEs) - have not adopted such

\footnotetext{
1,2 Department of Telecommunications and Media Informatics, Budapest University of Technology and Economics, Hungary

Email: \{franko,pvarga\}@tmit.bme.hu
}

state-of-the-art solutions (e.g., robots for logistical tasks) yet, since it would not be beneficial financially in the short term currently. Nonetheless, SMEs can implement new technologies more rapidly than bigger enterprises, therefore they will remain as the backbone of manufacturing supply chains after the digital transition too. Nonetheless, it is more challanging to adopt Industry 4.0 technologies for SMEs often due to financial reasons, therefore it is crucial to choose strategy, since an effective implementation can offer tremendous benefits [3] [4] [5]. Numerous researches investigated methods, strategies and roadmaps for adopting industry 4.0 technologies efficiently for SMEs, thus each related work - including this current article - can relevant for SMEs too [6] [7]. Due to this, there are ongoing researches that investigate the opportunities of utilizing machine learning in smart factories.

These researches mainly focus on (i) automating and optimizing maintenance tasks by monitoring machinery to provide intelligent fault detection and root cause analysis, (ii) predicting remaining useful life of a piece of equipment or tool, and (iii) automating quality management supported by computer vision and machine learning-based classification, creating automated production lines. This paper aims to compile a stateof-the-art survey on machine learning-based methods and solutions regarding smart maintenance and intelligent quality control as core tasks of smart manufacturing. It also describes potential challenges and research gaps of these fields to be solved, as well as challenges that are already addressed and hot research topics.

The paper is organized as follows: Section II gives a general overview of the role of machine learning in smart manufacturing and introduces maintenance and quality control as important tasks within this ecosystem. Section III and section IV investigate machine learning approaches, methods and algorithms regarding smart maintenance (diagnostics and prognostics) and intelligent quality control (non-visual and computer vision aided), respectively. Section V concludes the paper.

\section{OVERVIEW OF SMART MANUFACTURING ECOSYSTEM}

Manufacturing operations include numerous processes and tasks which have always been critical in terms of safety, reliability, and cost-efficiency. Hence, increasing control over these operations is a focal point in industrial digitization trends. Traditionally there are few fields considered the targets of industrial automation - such as assembly or manufacturing generally [8] - but nowadays, as smart factories emerging, these traditional automation targets have been started to share 
the spotlight with other fields such as maintenance, quality control, and many others. In this paper, the focus is on maintenance and quality control applications, however, these two fields cannot be fully discussed without mentioning related fields, which provide the input for these tasks, and which digitalization also has a significant impact on.

In the last few years, machine learning applications, especially deep learning for smart manufacturing, were investigated in order to discover the areas well-worth improving [10]. Generally, machine learning offers great potentials due to the intense presence of big data and thus opens new ways to develop advanced, data-driven applications [11]. Moreover, machine learning methods such as convolutional and recurrent neural networks, auto encoder, etc. [12] [13] allow us (i) to model complex systems with limited domain knowledge due to the feature extraction and also (ii) to optimize specific processes and tasks based on this model. It is often required to estimate performance and make decisions based on available input data. Authors of [14] presented the concept of a machine learning-based Design Support System (DSS) to address some sort of optimization problems in design processes regarding manufacturing, logistics, and warehouse management. Nonetheless, deep expert knowledge is still required since manufacturing data cannot be utilized in its raw form due to its high dimensions [15], thus regarding smart planning and design, the usage of knowledge-based models is still one of the most important methodologies.

As it is shown in Figure 1, both maintenance and quality control has strong connections with related subfields and tasks within smart manufacturing, such as the aforementioned Smart design and Smart planning. Due to being versatile, machine learning is commonly used within smart industry; therefore, the general research gaps and challenges are the same for these smart tasks. However, specific applications raise new problem sets as well as new solutions; thus, there are unique challenges and research gaps the can be identified. In the following chapters, machine learning applications will be investigated from the aspect of maintenance and quality control to give a comprehensive overview of the identified challenges, gaps, and hot research topics. The figure lists energy optimization or energy efficiency management as one of the goals of maintenance. However, it is a focal point of smart manufacturing; it is a specific task, therefore; in this article, this concept is not presented.

\section{MACHINE LEARNING IN SMART MAINTENANCE}

One of the most important tasks in each segment of the industry is maintenance. Unexpected malfunctions can lead to undesirable consequences such as stopping assembly lines or reschedule logistical processes, which cause economic loss directly or indirectly e.g., due to delays in operations.

The mechanism of maintenance seems to be simple; however, smart and efficient maintenance involves numerous tasks and each of them improves the efficiency of the overall mechanism. In its purest form, maintenance is reactive; thus, keeping certain tools, machines, or pieces of equipment in good condition is not a part of maintenance. So, in the case of reactive maintenance, these machines and tools will be repaired only after a breakdown and not earlier. Nevertheless, in certain cases, the occurrence of malfunctions is not obvious; therefore, the condition of machinery continues to deteriorate; thus, fault detection techniques are required to indicate the need for maintenance. Additionally, applying diagnostics and root cause analysis can enhance the quality of maintenance, especially when the source of failure is unknown and must be investigated.

Modern maintenance paradigms such as preventive, predictive, and proactive maintenance use different approaches than the reactive one. Preventive maintenance - as its name suggests - focuses on keeping the tools and machinery in good condition, which requires frequent examination of wearout level. Optimizing the efficiency of this paradigm by eliminating unnecessary examination and repairing is achieved by using telemetry, external sensors, and other condition monitoring techniques to collect data for diagnostics. Predictive maintenance also utilizes the tools above; however, it has a different purpose: to estimate the date of a breakdown so the whole process can be planned. For precise estimations, certain models of the monitored asset are used to calculate its remaining useful life (RUL) [17]. As proactive maintenance unified these two paradigms - predictive and preventive -the used toolsets were combined too, and with the rise of machine learning and Industrial Internet of Things (IIoT), it became a dynamic, data-driven approach.

Being data-driven also means that a massive amount of data is required, often provided by related smart tasks, processes, systems or even logs [18]. For example, the growing tendency in adapting technologies such MES as Plant floor automation and information systems (PES) can give prognostics-based maintenance a boost by generating a huge amount of data [19]. As an essential concept of smart manufacturing, the Digital Twin as a high-level, data-driven, abstract model of systems also eases data aggregation and analytics. It also improves the overall effectiveness of proactive maintenance, thus reducing the potential of failure [20].

\section{A. Diagnostics}

Diagnostics in smart maintenance usually consist of two main parts: fault detection (FD) and root cause analysis (RCA). Fault detection aims to monitor the machinery and indicate that if components or parts of the machine wear out or deteriorate, RCA investigates the malfunction to find the root cause.

Fault detection has always been a difficult task for learning systems due to the overly biased training data. Moreover, despite the numerous available high-performance learning algorithms, prior domain knowledge is required to implement effective fault detection and diagnosis (FDD) systems [21]. In this case, the aim of learning is to make the learning system detect anomalies successfully, and the obvious bottleneck is not having a sufficient number of samples that represent faults and malfunctions, since, in a well-maintained environment, such things are avoided as much as possible. In [22] Fathy et al. presented a comprehensive analysis of different techniques to deal with imbalanced data. Although their results showed 


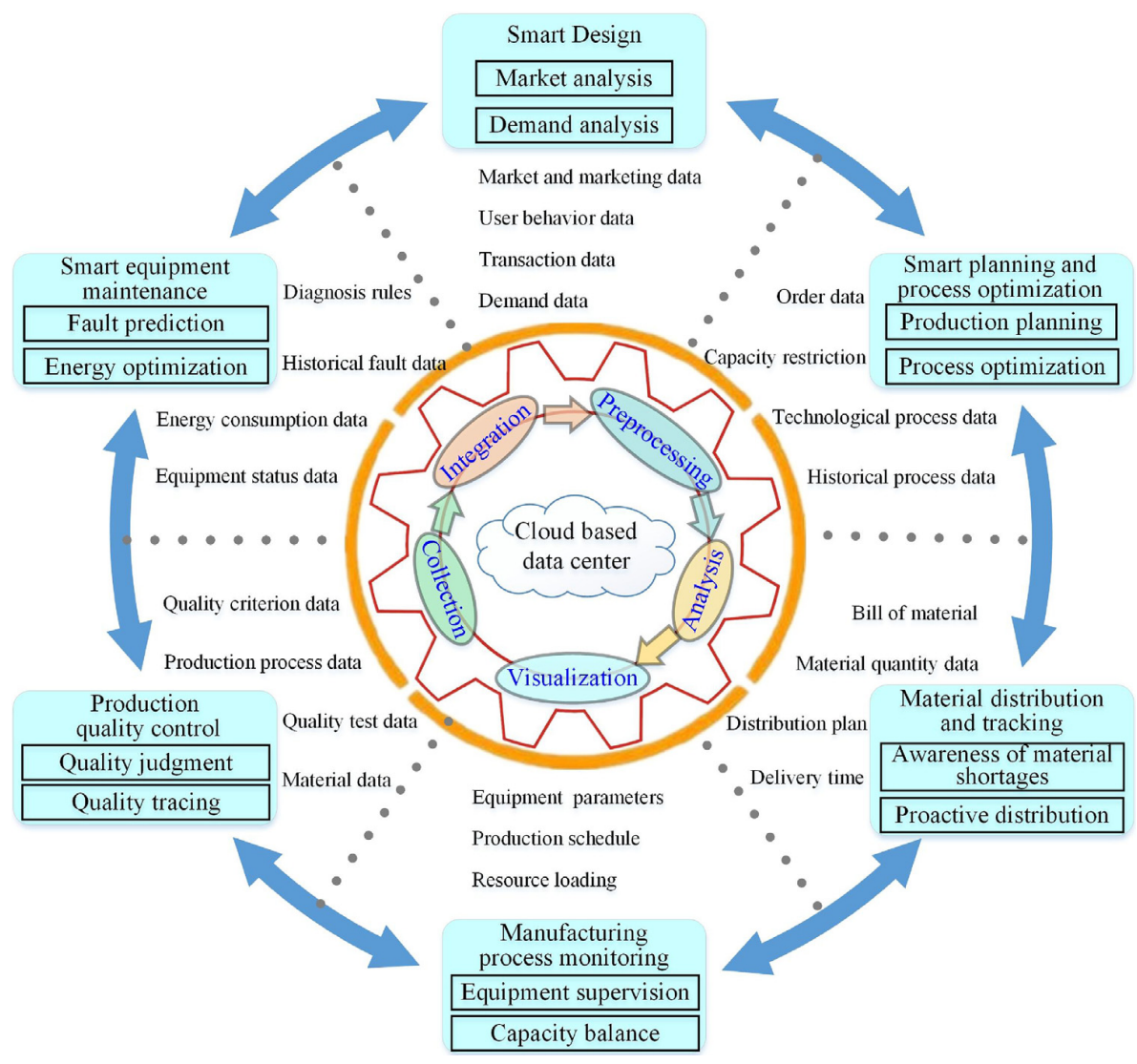

Fig. 1. The role of smart maintenance and smart quality control in a data-driven smart manufacturing ecosystem [9].

no one-solution-fits-all in binary classification, applying synthetic oversampling or generative adversarial networks (GAN) highly improves fault detection performance. Authors of [23] presented a deep learning-based FDD method that utilizes GAN to generate fake samples in order to balance and expand the training dataset and showed the performance of different methods for especially rotating machinery as a common type of equipment in manufacturing. Authors of [24] also sucessfully utilized a customzied GAN to handle imbalanced-class problem for fault detection diagnosis of chillers. In [25] an FDD method was introduced towards imbalanced data as well. However, the proposed new WMODA technique - based on synthetic oversampling - is limited to binary-class imbalanced data problems; it was demonstrated that WMODA performs better than several other imbalanced data learning methods and the traditional data-driven methods.

Another major drawback of ML-based fault detection applications is that a trained system can be used only for one specific machine, which is represented by the collected dataset [21]. It would be promising if similar systems with the same properties and characteristics by default could be trained by a common, unified dataset consisting of each collected sample from all involved systems. Transfer learning aims to solve this problem set. Lei et al. [26] address transfer learning as one of the most important research topics within MLbased, and big data enabled fault detection in the future.
It also highlights the importance of deep transfer learning, GAN, and Transfer factor analysis as methodologies capable of being used in transfer scenarios where data is collected from identical machines (TIM) as well as from different machines (TDM). In [27], authors propose a deep generative transfer learning system for fault diagnostics on new hard disk, and showed that this method outperfomed other ones in this application due to the low number of real faulty samples.

The other important task in diagnostics is the aforementioned root cause analysis, which is more of a collection of models and techniques that share the same basic principles and workflow as one straight algorithm or method. The chosen model depends on the problem set and the observed system since domain, or system knowledge may be required. Authors of [28] presented an exhaustive and detailed survey of applied RCA approaches, methods, and technologies, and a broad set of these can be seen in Figure 2. In [29] address two major issues regarding RCA, (i) the lack of supervised datasets and (ii) the need for interpretability to foster trust are addressed as major obstacles of intensive use of machine learning in anomaly detection; therefore, the authors propose random forest technique to enable using unlabeled data in root cause analysis.

However, there is much ongoing research in this field; these works mainly discuss topics from a general perspective rather than presenting actual applications - in contrast to the previous 


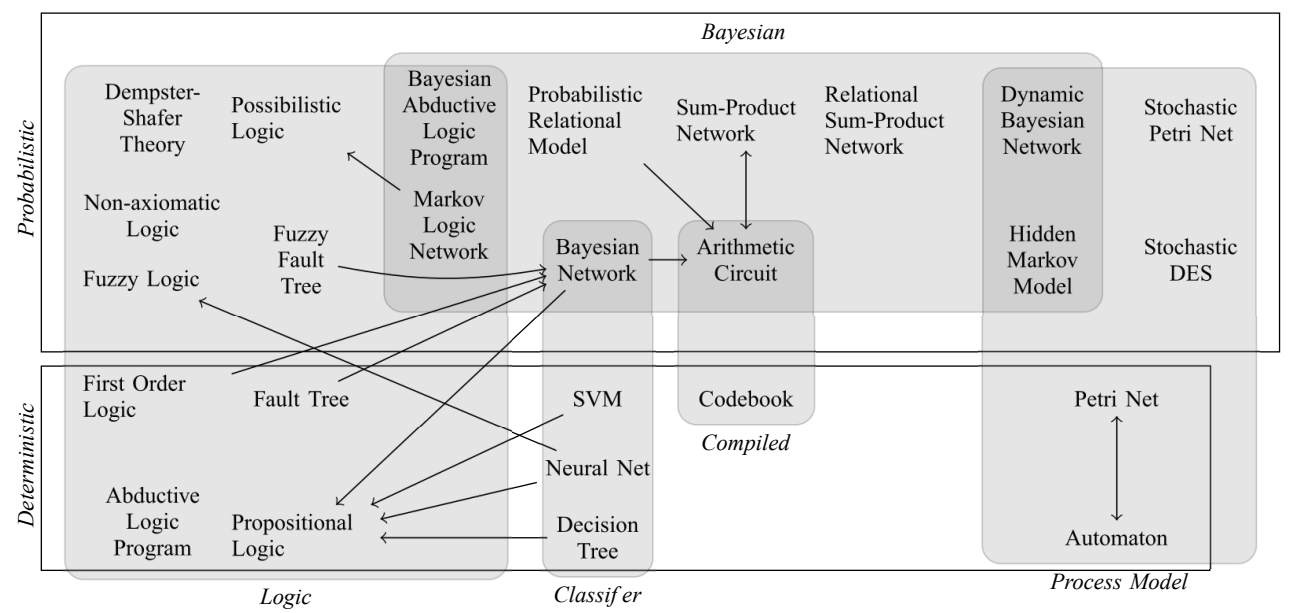

Fig. 2. Classification of models and methods that are used in Root Cause Analysis. Directed arrows indicate possible conversions between different models [16].

decade. Nonetheless the number of articles that utilize the aforementioned methods such as GANs or transfer learning in industrial use-cases is increasing as it is shown in table I.

\begin{tabular}{ccc}
\hline Citation & Application & Used method \\
\hline$[30]$ & Power plant thermal system & $\begin{array}{c}\text { transfer learning } \\
\text { (HDDA) } \\
\text { transfer learning } \\
\text { (many) }\end{array}$ \\
{$[31]$} & Rolling element bearings (train bogie) & $\begin{array}{c}\text { transfer learning } \\
\text { (deep generative) }\end{array}$ \\
{$[32]$} & Laboratory/Locomotive bearings & GAN \\
{$[33]$} & rolling bearings & GAN \\
{$[34]$} & Wind Turbines Gearbox & \\
\hline
\end{tabular}

TABLE I

CUTTING-EDGE SMART MAINTENANCE APPLICATIONS

\section{B. Prognostics, predictions and health management}

Prognostics and predictions - or as often called: predictions and health management (PHM) - play essential roles in smart maintenance since they enable precise and accurate scheduling maintenance tasks in an optimal manner. The basic concept includes constructing a model of the machine based on historical data and monitoring it in real-time, and predicting its future behavior by using real-time data as the input of the established model. This concept's main goal is to estimate the remaining useful life (RUL) of a tool or machinery as precisely as possible.

Making predictions is beneficial in such a field where machine learning techniques perform very well traditionally [35], since most predictions include different hidden factors that are challenging or impossible to investigate by other methods. Certain applications and researches involving prognostics to calculate RUL are related to medical sciences, healthcare, or health management, where the main targets of the estimation are patients or course of disease. Nevertheless, these results also come in handy for smart maintenance and often directly applicable in such systems.

Traditionally, there are three approaches that are widely used in a prognostic manner: physics/model-based, data- driven, and hybrid approaches [36]. In the last decade, the data-driven approach obviously dominated this field [37] [38] with mostly stochastic algorithms - most notably Bayesian networks - which and are still efficient in related fields or in safety use-case where the uncertainty of a solution has to be known [39] [40].

Regarding the applied technologies that are shown in Figure 3 , it is safe to say that a wide range of machine learning techniques and algorithms are used in prognostics that cover specific use-cases [41], depending on the environment, the input data, the level of domain or expert knowledge. Therefore in this topic, the main challenges of machine learning-based techniques are usually related to these aspects, such as data acquisition and handling big data in terms of scalability, latency, and network bandwidth, moreover finding machine learning applications that are suitable for multiple use-cases [42].

It is also worth mentioning that some of the issues addressed in other sections of this paper, such as dealing with unlabeled data [43] or flexibility of a solution [44] are also valid challenges for prognostics and health management. It goes for the same for future researches regarding transfer learning, generative algorithms, and physics-induced machine learning as well [45] [46].

\section{INTELLIGENT QUALITY CONTROL}

While proper maintenance ensures that the production line behaves and works as it should, quality control ensures that the final product meets different (inner and outer) standards. Quality control is as important, if not more so, than maintenance, since if quality control fails, poor or inadequate products will be shipped for the customers.

The first big, revolutionary step in quality control was when the application of statistical methods, such as control charts and acceptance sampling, had begun. Since then, the theory of quality management and quality control has been evolving by creating new approaches and paradigms that fuel the automating intentions within the industry towards quality control processes. In this topic, the machine learning applications are very heterogenous since they include regular 


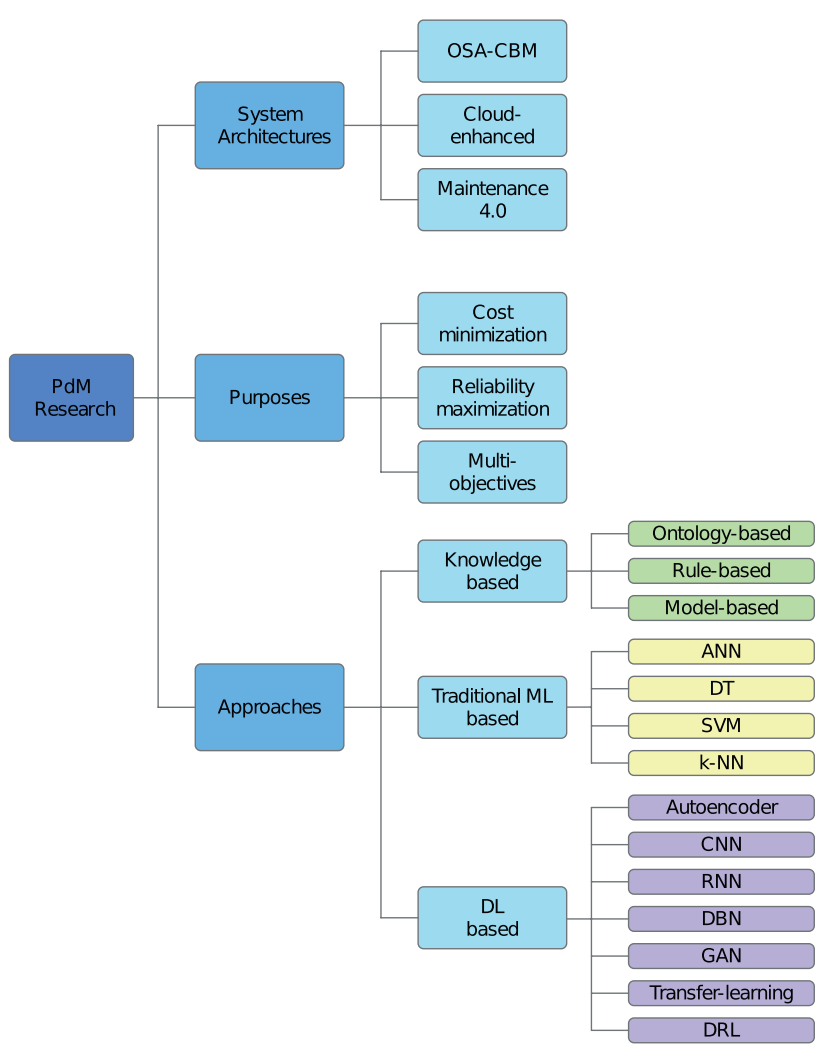

Fig. 3. Taxonomy of predictive maintenance including the applied technologies and models [47]

classification based on collected data, but also computer vision and image recognition.

As Cyber-physical production systems (CPPS) and IIoT became prevalent paradigms within the industry, they enabled such data-intensive applications like the aforementioned digital twin concept. In [48] the authors proposed a new Digital Twin modeling method based on Multi-agent System (MAS) and semantic models that provide improved quality control during the manufacturing phase of the product. Authors of [49] also introduced a digital twin-based quality control solution while emphasizing that it is strongly coupled to assembly and production line optimization.

From the technical aspect, smart maintenance and smart quality control might seem analogous, so in most cases, problems can be formulated in the same way: e.g., monitoring and quality inspection of machinery can be implemented by using the same approach and technologies that would be used in quality insurance or quality control of products. This would imply that all the solutions above for smart maintenance could be applied for quality control; however, these tasks have different requirements and characteristics than maintenance tasks.

While smart maintenance deals mostly with inspecting and monitoring a static, single piece of equipment, quality inspection covers the investigation and classification of great quantities of products that change dynamically. Consequently, the cited smart maintenance design approaches and technolo- gies are also frequently used for quality control applications, but this field must be discussed separately since its unique requirements. Moreover, it is worth noting that quality control applications usually rely upon domain knowledge-based defect detection architectures, design approaches, and solutions.

\section{A. Non-visual quality control applications}

This approach of quality control, which involves machine learning applications - mostly deep neural networks, but not computer vision - relies upon telemetry data and datasets that are measured and gathered by sensors across the whole assembly or production line [50]. As with all similar machine learning solutions, this one also requires a huge amount of data in the training phase; therefore, the implemented system will be rigid since each time the production line changes, the model has to be retrained again. While computer visionbased solutions offer more flexibility, this approach is fast, reliable, and considered lightweight. Moreover, techniques like Lifelong Learning with Deep neural networks (L-DNN) [51] can enhance the flexibility of such a quality control system, creating an alternative to image recognition-based solutions that can be considered lightweight.

Most of the quality control application that uses machine learning formalize the problem as binary classification, e.g., pass or deny. However, certain cases require more complex formalization; therefore, the designed architecture, the applied learning algorithms, and pattern recognition strategies often vary. In [52] an $l_{1}$ regularized logistic regression is used as a learning algorithm in order to create a system that can successfully detect rare quality events to achieve defect-free processes. Authors of [52] also presented two use-cases, where different model selection criteria were applied to find the optimal one among multiple ones, which were created as a result of manually defining features. It has to be emphasized that regarding machine learning-based quality control applications, there are no one-size-fits-all approaches, algorithms, or technologies because the characteristics of the production line have a huge impact on the system to be implemented.

The properties and characteristics of the proposed solutions can be very diverse depending on the applied architecture, algorithm, approaches, and other building blocks of such systems. However, the accuracy of classification is one of the most important properties; availability, reliability, performance, response time, and resource requirements play key roles in choosing the appropriate solution for a specific task, as always.

Authors of [53] investigate several binary classification models for quality control of Multistage Manufacturing Process (MMP) and measured the performance of different algorithms, namely: Gaussian Naive Bayes (GNB), K-Nearest Neighbours (KNN), XGBOOST, Random Forest, and Support Vector Machine (SVM). Since in MMP an early stage defect or failure will propagate, it is essential to detect these defects as soon and fast as possible, however; the quality inspection problem of each stage is less complex than the final one. In [53] it was shown, the aforementioned algorithms performed very well in an MMP; while they are considered lightweight 
in contrast to any neural networks, therefore they can be efficiently applied at each stage of MMP to inspect production quality.

In [54] a Bi-LSTM (Long-Short Term Memory) based solution was proposed, where the implemented system uses control charts and histograms as input data to find patterns; therefore, it is an indirect approach, which uses statistical learning. In this case, it is easier to define feature sets for recognizing histogram and control chart patterns than using raw data as input; therefore, this solution can be considered lightweight.

When it comes to performance - and scalability in most cases - where lightweight solutions can not be utilized, cloud computing is an effective and usually cost-efficient way to deal with heavy resource issues [55]. Nowadays, almost every cloud provider offers services for machine learning applications; moreover, these can be connected to other cloud-based applications such as warehouse management, etc. [56]. It can often be the optimal solution if the performance must be improved, although in this case, the quality of the connection will be the bottleneck. Authors of [57] introduce a combined edgecloud-based quality inspection system that trains the model in the cloud to enable high-performance in training phase, but it deploys the model in the edge; thus, the response time will be much faster compared to an only cloud-based one.

\section{B. Computer vision-aided quality control}

Another technique regarding machine learning-aided quality control is the computer vision $(\mathrm{CV})$ based quality inspection. However, CV-enabled feature extraction is not a fresh field; it is still a hot topic since it has not been adopted by industrial players as fast as it was expected. Nevertheless, this technology is used more widely than the one mentioned above since it is suitable for quality control of non-manufactured products (e.g., food) that can not be monitored one by one via sensors.

This method is much more flexible than the previous one since it can handle complex, complicated, non-binary classification problems while being robust. Moreover, it can be applied in dynamic environments that often change because it is contactless; while numerous methods based on sensing require some sort of physical interaction, therefore they are less flexible or not flexible at all. Nonetheless, image processing is almost always slower than applying binary classification (including inference and training time) purely based on sensor or telemetric data; therefore, the applicability of this technique in the case of fast, high-throughput assembly or production lines is limited [58], [59].

The flexibility and performance of such a system depend on the applied technology and the followed approaches while designing the architecture. The most important factors are usually the features that will be examined during the investigation of an object to find defects; therefore, defining features is a crucial task of designing an optical quality control (OQC) system. Another approach to design fast and reliable OQC systems is applying Convolutional Neural Networks $(\mathrm{CNN})$ that provide automatic feature extraction instead of the aforementioned manual one. In [58] Weimer et al. showed that CNN-based architectures are capable of identifying defects with high accuracy; therefore, it is possible to create reliable OQC systems with limited prior knowledge on the problem domain. Authors of [60] presented a CNN-based OQC system with unique architecture to find the optimal accuracy and training time. Therefore they limited the number of the used convolutional layer. They showed that an optimum exists for this specific task, which has better (or similar) accuracy than other CNN architecture while its training time is considerably faster.

However, image processing is usually a computation heavy task; therefore, in certain cases, it can be beneficial to apply prior knowledge to increase the system's performance. The authors of [59] designed and implemented a Convolutional Neural Network-based quality control system for PET bottle caps, which system is supported by image calibration to enable using a custom, lightweight architecture. In spite of that, not so customizable $\mathrm{CV}$ frameworks that enable fast prototyping are extremely popular in OQC systems: e.g., a well-known one is the "You only look once" (YOLOv3) [61]. YOLOv3 provides impressive performance with appropriate detection accuracy and very fast inference time, although it is not the best-performing choice compared to the previous one.

\section{CONCLUSION}

In this paper, we investigated machine learning-based applications within smart maintenance and intelligent quality control that are core tasks of smart manufacturing while identifying the most important application-specific challenges and research gaps.

Regarding smart maintenance applications: (i) synthetic oversampling, generative adversarial networks, and (ii) transfer learning have been identified as hot research topics that address the problem of (i) imbalanced, overly biased data and (ii) using the same dataset for other different or identical machines. The lack of supervised datasets is a common issue for RCA, IDF, prognostics, and many fields, which may lose its importance as smart manufacturing becomes more mature. However, right now, the best practice is using such an approach the can easily deal with unlabeled data, e.g., random forest. So, the field of applications with unlabeled data is identified as a hot topic as well, since in this early-mid phase of smart factory development, using unlabeled data set is a common scenario.

In the field of quality control, both non-visual and computer vision-aided applications have a great reputation. Non-visual solutions are lightweight CV-based solutions that are much more flexible, while both of them perform very well. The main challenges for non-visual quality control applications are: enabling high-data-rate and enhance flexibility to enable quality control in the ever-changing smart factories. The main challenges for CV-based applications are: improve the inference time and decrease the required computational capacity to enable image recognition-based quality inspection in highthroughput assembly and production lines. 


\section{REFERENCES}

[1] DIN Std., Reference Architecture Model Industrie 4.0 (RAMI4.0), DIN SPEC 91345, 042016.

[2] J. P. Usuga Cadavid, S. Lamouri, B. Grabot, R. Pellerin, and A. Fortin, "Machine learning applied in production planning and control: a state-of-the-art in the era of industry 4.0," Journal of Intelligent Manufacturing, 01 2020, DoI: 10.1007/s 10845-019-01531-7.

[3] D. Matt, V. Modrák, and H. Zsifkovits, Industry 4.0 for SMEs Challenges, Opportunities and Requirements: Challenges, Opportunities and Requirements, 01 2020, DOI: 10.1007/978-3-030-25425-4.

[4] S. Menon and S. Shah, "Are smes ready for industry 4.0 technologies: An exploratory study of i 4.0 technological impacts," in 2020 International Conference on Computation, Automation and Knowledge Management (ICCAKM), 2020, pp. 203-208, DoI: $10.1109 /$ ICCAKM46823.2020.9051550.

[5] E. Sezer, D. Romero, F. Guedea, M. Macchi, and C. Emmanouilidis, "An industry 4.0-enabled low cost predictive maintenance approach for smes," in 2018 IEEE International Conference on Engineering, Technology and Innovation (ICE/ITMC), 2018, pp. 1-8, DoI: $10.1109 /$ ICE.2018.8436307.

[6] A. Cotrino, M. A. Sebastián, and C. González-Gaya, "Industry 4.0 roadmap: Implementation for small and medium-sized enterprises," Applied Sciences, vol. 10, no. 23, 2020. [Online]. Available: https:// www.mdpi.com/2076-3417/10/23/8566. DoI: 10.3390/app10238566

[7] T. Taurino and A. Villa, "A method for applying industry 4.0 in small enterprises," IFAC-PapersOnLine, vol. 52, no. 13, pp. 439-444, 2019. 9th IFAC Conference on Manufacturing Modelling, Management and Control MIM 2019. [Online]. Available: https://www.sciencedirect. com/science/article/pii/S2405896319310407.

DoI: 10.1016/j.ifacol.2019.11.099

[8] R. Seidel, A. Mayr, F. Schäfer, D. Kißkalt, and J. Franke, "Towards a smart electronics production using machine learning techniques," in 201942 nd International Spring Seminar on Electronics Technology (ISSE), 2019, pp. 1-6, DoI: 10.1109/ISSE.2019.8810176.

[9] F.Tao,Q.Qi,A.Liu, and A.Kusiak, "Data-drivensmartmanufacturing," Journal of Manufacturing Systems, vol. 48, pp. 157-169, 2018, special Issue on Smart Manufacturing. [Online]. Available: https:// www.sciencedirect.com/science/article/pii/S0278612518300062. DOI: $10.1016 /$ j.jmsy.2018.01.006

[10] M. Sharp, R. ak, and T. Hedberg, "A survey of the advancing use and development of machine learning in smart manufacturing," Journal of Manufacturing Systems, vol. 48,03 2018, DOI: $10.1016 /$ j.jmsy.2018.02.004

[11] C.Y.Park, J.W. Kim, B. Kim, and J. Lee, "Prediction for manufacturing factors in a steel plate rolling smart factory using data clusteringbased machine learning," IEEE Access, vol. 8, pp. 60 890-60 905, 2020, DoI: 10.1109/ACCESS.2020.2983188.

[12] J. Wang, Y. Ma, L. Zhang, R. X. Gao, and D. Wu, "Deep learning for smart manufacturing: Methods and applications," Journal of Manufacturing Systems, vol. 48, pp. 144-156, 2018, special Issue on Smart Manufacturing, DoI: 10.1016/j.jmsy.2018.01.003.

[13] R. Iqbal, T. Maniak, F. Doctor, and C. Karyotis, "Fault detection and isolation in industrial processes using deep learning approaches," IEEE Transactions on Industrial Informatics, vol. 15, no. 5, pp. 30773084, 2019, DOI: 10.1109/TII.2019.2902274.

[14] L. Romeo, J. Loncarski, M. Paolanti, G. Bocchini, A. Mancini, and E. Frontoni, "Machine learning-based design support system for the prediction of heterogeneous machine parameters in industry 4.0," $E x$ pert Systems with Applications, vol. 140, p. 112869, 082019 , DoI: 10.1016/j.eswa.2019.112869.

[15] B. Chen, J. Wan, L. Shu, P. Li, M. Mukherjee, and B. Yin, "Smart factory of industry 4.0: Key technologies, application case, and challenges," IEEE Access, vol. 6, pp. 6505-6519, 2018, DoI: 10.1109/ACCESS.2017.2783682.

[16] M. Solé, V. Muntés-Mulero, A. I. Rana, and G. Estrada, "Survey on models and techniques for root-cause analysis," 2017.

[17] J. Sikorska, M. Hodkiewicz, and L. Ma, "Prognostic modeling options for remaining useful life estimation by industry," Mechanical Systems and Signal Processing, vol. 25, pp. 1803-1836, 12 2011, DOI: $10.1016 /$ j.ymssp.2010.11.018.

[18] M.Maurer,A. Festl, B. Bricel, G. Schneider, and M. Schmeja, "Automl for log file analysis (alfa) in a production line system of systems pointed towards predictive maintenance," Infocommunications Journal, vol. 13, no. 3, pp. 76 - 84, 2021, DoI: 10.36244/ICJ.2021.3.8.
[19] S. T. Bukkapatnam, K. Afrin, D. Dave, and S. R. Kumara, "Machine learning and ai for long-term fault prognosis in complex manufacturing systems," CIRP Annals, vol. 68, no. 1, pp. 459-462, 2019, DoI: 10.1016/j.cirp.2019.04.104.

[20] A. Oluwasegun and J.-C. Jung, "The application of machine learning for the prognostics and health management of control element drive system," Nuclear Engineering and Technology, vol. 52, no. 10, pp. 2262-2273, 2020. [Online]. Available: https://www.sciencedirect. com/science/article/pii/S1738573319308654 DOI: $10.1016 /$ j.net.2020.03.028

[21] S. R. Saufi,Z.A. B. Ahmad, M. S. Leong, and M. H. Lim, "Challenges and opportunities of deep learning models for machinery fault detection and diagnosis: A review," IEEE Access, vol. 7, pp. 122644 122662, 2019, DOI: 10.1109/ACCESS.2019.2938227.

[22] Y. Fathy, M. Jaber, and A. Brintrup, "Learning with imbalanced data in smart manufacturing: A comparative analysis," IEEE Access, vol. PP, 12 2020, DoI: 10.1109/ACCESS.2020.3047838.

[23] W. Zhang, X. Li, X.-D. Jia, H. Ma, Z. Luo, and X. Li, "Machinery fault diagnosis with imbalanced data using deep generative adversarial networks," Measurement, vol. 152, p. 107377, 2020. [Online]. Available: https://www.sciencedirect.com/science/article/pii/ S0263224119312412. DOI: 10.1016/j.measurement.2019.107377

[24] K. Yan, A. Chong, and Y. Mo, "Generative adversarial network for fault detection diagnosis of chillers," Building and Environment, vol. 172, p. 106698, 2020. [Online]. Available: https://www.sciencedirect. com/science/article/pii/S0360132320300561 DoI: $10.1016 /$ j.buildenv.2020.106698

[25] Y. Zhang, X. Li, L. Gao, L. Wang, and L. Wen, "Imbalanced data fault diagnosis of rotating machinery using synthetic oversampling and feature learning," Journal of Manufacturing Systems, vol. 48, pp. 34-50, 2018, special Issue on Smart Manufacturing. [Online]. Available: https:// www.sciencedirect.com/science/article/pii/S027861251830044X. DOI: $10.1016 /$ j.jmsy.2018.04.005

[26] Y.Lei, B. Yang, X. Jiang, F. Jia, N. Li, and A. K. Nandi, “Applications of machine learning to machine fault diagnosis: A review and roadmap," Mechanical Systems and Signal Processing, vol. 138, p. 106587, 2020 [Online]. Available: https://www.sciencedirect.com/science/article/pii/ S0888327019308088. DOI: 10.1016/j.ymssp.2019.106587

[27] C. Shi, Z. Wu, X. Lv, and Y. Ji, "Dgtl-net: A deep generative transfer learning network for fault diagnostics on new hard disks," Expert Systems with Applications, vol. 169, p. 114379, 2021. [Online]. Available: https:// www.sciencedirect.com/science/article/pii/ S0957417420310551. DoI: $10.1016 /$ j.eswa.2020.114379

[28] M. Solé, V. Muntés-Mulero, A. I. Rana, and G. Estrada, "Survey on models and techniques for root-cause analysis," 2017. [Online]. Available: https://arxiv.org/abs/1701.08546

[29] M. Carletti, C. Masiero, A. Beghi, and G. A. Susto, "Explainable machine learning in industry 4.0: Evaluating feature importance in anomaly detection to enable root cause analysis," in 2019 IEEE International Conference on Systems, Man and Cybernetics (SMC), 2019, pp. 21-26, DoI: 10.1109/SMC.2019.8913901.

[30] X. Wang, H. He, and L. Li, "A hierarchical deep domain adaptation approach for fault diagnosis of power plant thermal system," IEEE Transactions on Industrial Informatics, vol. 15, no. 9, pp. 5139-5148, 2019, DOI: 10.1109/TII.2019.2899118.

[31] X. Li, W. Zhang, and Q. Ding, "Cross-domain fault diagnosis of rolling element bearings using deep generative neural networks," IEEE Transactions on Industrial Electronics, vol. 66, no. 7, pp. 55255534, 2019, DoI: 10.1109/TIE.2018.2868023.

[32] B. Yang, Y. Lei, F. Jia, and S. Xing, "An intelligent fault diagnosis approach based on transfer learning from laboratory bearings to locomotive bearings," Mechanical Systems and Signal Processing, vol. 122, pp. 692-706, 2019. [Online]. Available: https://www. sciencedirect.com/science/article/pii/S0888327018308367. DOI: $10.1016 / j . y m s s p .2018 .12 .051$

[33] F. Zhou, S. Yang, H. Fujita, D. Chen, and C. Wen, "Deep learning fault diagnosis method based on global optimization gan for unbalanced data," Knowledge-Based Systems, vol. 187, p. 104837, 2020. [Online]. Available: https://www.sciencedirect.com/science/article/pii/ S0950705119303120. DOI: 10.1016/j.knosys.2019.07.008

[34] X. Zhang, P. Wu, J. He, S. Lou, and J. Gao, "A gan based fault detection of wind turbines gearbox," in 2020 7th International Conference on Information, Cybernetics, and Computational Social Systems (ICCSS), 2020, pp. 271-275, DoI: 10.1109/ICCSS52145.2020.9336899. 
[35] C. Cheng, G. Ma, Y. Zhang, M. Sun, F. Teng, H. Ding, and Y. Yuan, "A deep learning-based remaining useful life prediction approach for bearings," IEEE/ASME Transactions on Mechatronics, vol. 25, no. 3 , pp. 1243-1254, 2020, DoI: 10.1109/TMECH.2020.2971503.

[36] Y. Wang, Y. Zhao, and S. Addepalli, "Remaining useful life prediction using deep learning approaches: A review," Procedia Manufacturing, vol. 49, pp. 81-88, 2020, proceedings of the 8th International Conference on Through-Life Engineering Services - TESConf 2019. [Online]. Available: https://www.sciencedirect.com/science/article/pii/ S2351978920316528. DOI: 10.1016/j.promfg.2020.06.015

[37] R. K. Singleton, E. G. Strangas, and S. Aviyente, "Extended kalman filtering for remaining-useful-life estimation of bearings," IEEE Transactions on Industrial Electronics, vol. 62, no. 3, pp. 1781-1790, 2015, DOI: 10.1109/TIE.2014.2336616.

[38] R. Khelif, B. Chebel-Morello, S. Malinowski, E. Laajili, F. Fnaiech, and N. Zerhouni, "Direct remaining useful life estimation based on support vector regression," IEEE Transactions on Industrial Electronics, vol. 64, no. 3, pp. 2276-2285, 2017, DoI: 10.1109/TIE.2016.2623260.

[39] C. HU, H. PEI, Z. WANG, X. SI, and Z. ZHANG, "A new remaining useful life estimation method for equipment subjected to intervention of imperfect maintenance activities," Chinese Journal of Aeronautics, vol. 31, no. 3, pp. 514-528, 2018. [Online]. Available: https://www. sciencedirect.com/science/article/pii/S1000936118300256. DOI: 10.1016/j.cja.2018.01.009

[40] M. Benker,L.Furtner,T. Semm, and M.F.Zaeh, "Utilizing uncertainty information in remaining useful life estimation via bayesian neural networks and hamiltonian monte carlo," Journal of Manufacturing Systems, 2020. [Online]. Available: https://www.sciencedirect.com/ science/article/pii/S0278612520301928. DOI: $10.1016 /$ j.jmsy.2020.11.005

[41] Z.M. Çınar, A. Abdussalam Nuhu, Q.Zeeshan, O. Korhan, M. Asmael, and B. Safaei, "Machine learning in predictive maintenance towards sustainable smart manufacturing in industry 4.0," Sustainability, vol. 12, no. 19, 2020. [Online]. Available: https://www.mdpi.com/20711050/12/19/8211. DoI: $10.3390 /$ su12198211

[42] J. Dalzochio, R. Kunst, E. Pignaton, A. Binotto, S. Sanyal, J. Favilla, and J. Barbosa, "Machine learning and reasoning for predictive maintenance in industry 4.0: Current status and challenges," Computer in Industry, vol. 123, p. 103298, 2020. [Online]. Available: https:// www.sciencedirect.com/science/article/pii/S0166361520305327. DOI: $10.1016 /$ j.compind.2020.103298

[43] A. Listou Ellefsen, E. Bjørlykhaug, V. Æsøy, S. Ushakov, and H. Zhang, "Remaining useful life predictions for turbofan engine degradation using semi-supervised deep architecture," Reliability Engineering \& System Safety, vol. 183, pp. 240-251, 2019. [Online]. Available: $\quad$ https://www.sciencedirect.com/science/article/pii/ S0951832018307506. DoI: $10.1016 /$ j.ress.2018.11.027

[44] M. Kraus and S. Feuerriegel, "Forecasting remaining useful life: Interpretable deep learning approach via variational bayesian inferences," Decision Support Systems, vol. 125, p. 113100, 2019. [Online]. Available: https://www.sciencedirect.com/science/article/pii/ S0167923619301290. DoI: $10.1016 /$ j.dss.2019.113100

[45] O. Fink, Q. Wang, M. Svensen, P. Dersin, W.-J. Lee, and M. Ducoffe, "Potential, challenges and future directions for deep learning in prognostics and health management applications," Engineering Applications of Artificial Intelligence, vol. 92, p. 103678, 06 2020, DOI: $10.1016 / j$.engappai.2020.103678.

[46] A. Diez-Olivan, J. Del Ser, D. Galar, and B. Sierra, "Data fusion and machine learning for industrial prognosis: Trends and perspectives towards industry 4.0," Information Fusion, vol. 50, 102018 , DoI: 10.1016/j.inffus.2018.10.005

[47] Y. Ran, X. Zhou, P. Lin, Y. Wen, and R. Deng, "A survey of predictive maintenance: Systems, purposes and approaches," 2019.

[48] X. Zheng, F. Psarommatis, P. Petrali, C. Turrin, L. Jinzhi, and D. Kiritsis, "A quality-oriented digital twin modelling method for manufacturing processes based on a multi-agent architecture," vol. 51, 06 2021, DOI: 10.1016/j.promfg.2020.10.044.

[49] Y. Ma, H. Zhou, H. He, G. Jiao, and S. Wei, "A digital twin-based approach for quality control and optimization of complex product assembly," in 2019 International Conference on Artificial Intelligence and Advanced Manufacturing (AIAM), 2019, pp. 762-767, DoI: 10.1109/AIAM48774.2019.00157.
[50] M. Miškuf and I.Zolotová, "Comparison between multi-class classifiers and deep learning with focus on industry 4.0," in 2016 Cybernetics Informatics (KI), 2016, pp. 1-5, DoI: 10.1109/CYBERI.2016.7438633.

[51] G. Parisi, R. Kemker, J. Part, C. Kanan, and S. Wermter, "Continual lifelong learning with neural networks: A review," Neural Networks, vol. 113, pp. 54-71, 02 2019, DoI: 10.1016/j.neunet.2019.01.012.

[52] C. Escobar and R. Morales-Menendez, "Machine learning techniques for quality control in high conformance manufacturing environment," Advances in Mechanical Engineering, vol. 10, p. 168781401875551, 02 2018, Dor: 10.1177/1687814018755519.

[53] R.S.Peres,J.Barata,P.Leitao, and G. Garcia, "Multistage quality control using machine learning in the automotive industry," IEEE Access, vol. 7, pp. 79 908-79 916, 2019, Dor: 10.1109/ACCESS.2019.2923405.

[54] T. Zan, Z. Liu, Z. Su, M. Wang, X. Gao, and D. Chen, "Statistical process control with intelligence based on the deep learning model," Applied Sciences, vol. 10, no. 1, 2020. [Online]. Available: https:// www.mdpi.com/2076-3417/10/1/308

[55] H. Li, K. Ota, and M. Dong, "Learning iot in edge: Deep learning for the internet of things with edge computing," IEEE Network, vol. 32, no. 1, pp. 96-101, 2018, DoI: 10.1109/MNET.2018.1700202.

[56] "Aws smart factory - manufacturing operations in the cloud," 2021.[Online]. Available: https://aws.amazon.com/manufacturing/smart-factory/

[57] J. Schmitt, J. Bönig, T. Borggräfe, G. Beitinger, and J. Deuse, "Predictive model-based quality inspection using machine learning and edge cloud computing," Advanced Engineering Informatics, vol. 45, p. 101101, 2020. [Online]. Available: https://www.sciencedirect.com/science/article/ pii/S1474034620300707. DoI: 10.1016/j.aei.2020.101101

[58] "Design of deep convolutional neural network architectures for automated feature extraction in industrial inspection," CIRP Annals, vol. 65, no. 1, pp. 417-420, 2016, DoI: 10.1016/j.cirp.2016.04.072.

[59] M. Malesa and P. Rajkiewicz, "Quality control of pet bottles caps with dedicated image calibration and deep neural networks," Sensors, vol. 21, p. 501, 01 2021, DoI: 10.3390/s21020501.

[60] J. Villalba-Diez, D. Schmidt, R. Gevers, J. Ordieres-Meré, M. Buchwitz, and W. Wellbrock, "Deep learning for industrial computer vision quality control in the printing industry 4.0," Sensors, vol. 19, no. 18, 2019. [Online]. Available: https://www.mdpi.com/1424-8220/ 19/18/3987. DOI: $10.3390 /$ s19183987

[61] J. Redmon, S. Divvala, R. Girshick, and A. Farhadi, "You only look once: Unified, real-time object detection," in 2016 IEEE Conference on Computer Vision and Pattern Recognition (CVPR), 2016, pp. 779788, DoI: $10.1109 /$ CVPR.2016.91.

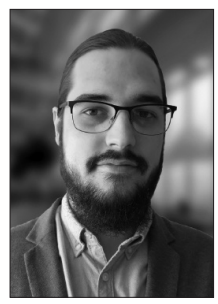

Attila Frankó received his M.Sc. degree in 2019 in Electrical Engineering from the Budapest University of Technology and Economics, Hungary. He is currently working on his Ph.D. degree at the Department of Telecommunications and Media Informatics. His research interests include Industrial Internet of Things especially machine learning based smart maintenance, logistic applications, cooperative Systems and, lowpowered security for wireless applications.

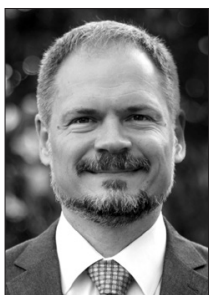

Pal Varga received his Ph.D. degree from the Budapest University of Technology and Economics, Hungary. $\mathrm{He}$ is currently an Associate Professor at the Budapest University of Technology and Economics and also the Director at AITIA International Inc. His main research interests include communication systems, Cyber-Physical Systems and Industrial Internet of Things, network traffic analysis, end-to-end QoS and SLA issues - for which he is keen to apply hardware acceleration and artificial intelligence, machine learning techniques as well.

Besides being a member of HTE, he is a senior member of IEEE, where he is active both in the IEEE ComSoc (Communication Society) and IEEE IES (Industrial Electronics Society) communities. He is Editorial Board member of the Sensors (MDPI) and Electronics (MDPI) journals, and the Editor-in-Chief of the Infocommunications Journal. 